\title{
Consistent verbal labels promote odor category learning
}

\section{Author 1 (corresponding)}

Name: Norbert Vanek

Affiliation: University of York

E-mail: norbert.vanek@york.ac.uk

Telephone: 00441904323951

Address: Centre for Research in Language Learning and Use, Department of Education, University of York, York, YO10 5DD, United Kingdom

ORCID: https://orcid.org/0000-0002-7805-184X

\section{Author 2}

Name: Márton Sóskuthy

Affiliation: University of British Columbia

E-mail: marton.soskuthy@ubc.ca

ORCID: https://orcid.org/0000-0002-5074-4767

\section{Author 2}

Name: Asifa Majid

Affiliation: University of York

E-mail: asifa.majid@york.ac.uk

ORCID: https://orcid.org/0000-0003-0132-216X

\section{Keywords}

odor categorization, perceptual learning, linguistic relativity, olfactory cognition

Author Note: Research reported in this study was funded by a Pump Priming Grant awarded to Norbert Vanek by the Department of Education, University of York, and by a start-up grant awarded to Asifa Majid by the Department of Psychology, University of York. We thank Alice Cruickshank for her help during experiment setup, and Haoruo Zhang for her assistance with testing.

Conflict of Interest Statement: The authors declare that the research was conducted in the absence of any potential conflict of interest 


\begin{abstract}
Recent research shows that speakers of most languages find smells difficult to abstract and name. Can verbal labels enhance the human capacity to learn smell categories? Few studies have examined how verbal labeling might affect non-visual cognitive processes, and thus far very little is known about word-assisted odor category learning. To address these gaps, we tested whether different types of training change learning gains in odor categorization. After four intensive days of training to categorize odors that were copresented with arbitrary verbal labels, people who learned odor categories with odor-label pairs that were more consistent were significantly more accurate than people with the same perceptual experience but who had odor-label pairs that were less consistent. Both groups' accuracy scores improved, but the learning curves differed. The context of consistent linguistic cuing supported a steady increase in correct responses from the onset of training. However, inconsistent linguistic cuing delayed the start of approximating to target odor categorization. These results show that associations formed between odors and novel verbal labels facilitate the formation of odor categories. We interpret this as showing a causal link between language and olfactory perceptual processing in supporting categorization.
\end{abstract}

\title{
1. Introduction
}

One unresolved source of controversy in cognitive science is the power of language to influence people's categorical perception of sensory input. In the domain of vision, evidence for, as well all against, language-specific effects often comes from crosslinguistic studies on the categorical perception of objects (e.g., Boutonnet, Dering, Viñas-Guasch, \& Thierry, 2013 vs. Malt, Sloman, Gennari, Shi, \& Wang, 1999), colors (e.g., Thierry, Athanasopoulos, Wigget, Dering, \& Kuipers, 2009 vs. Franklin, Pilling, \& Davies, 2005), and motion events (e.g., Athanasopoulos, Bylund, Montero-Melis, Damjanovic, L. et al., 2015 vs. Papafragou, Hulbert, \& Trueswell, 2008). Critics argue that attributing language effects, or their absence, to results of tests with speakers of different natural languages is problematic because possible confounding factors such as variation in people's culture-specific experiences or in previous knowledge cannot be excluded (e.g. Freundlieb, Ridder, Dobel, Enriquez-Geppert, et al., 2012). These potential confounds can be eliminated by training the same group of speakers to categorize familiar concepts with consistently or inconsistently paired novel verbal labels (e.g. Lupyan \& Ward, 2007), for instance, or even better, combining new verbal labels with unfamiliar or difficult-to-name concepts (e.g., using tactile stimuli, Miller, Schmidt, Blankenburg, \& Pulvermüller, 2019).

In this study, English native speakers were trained to categorize odors which were consistently or inconsistently paired with novel words. The aim was to test two alternative theoretical accounts that would predict distinct outcomes. Under the "universalist" view (e.g., Gleitman \& Papafragou, 2005; Jackendoff, 1990), one would expect no language effect on the formation of odor categories because categorization operates independently from linguistic encoding. In contrast, "relativist" or "constructionist" accounts (e.g. Boroditsky, 2001; Bowerman \& Levinson, 2001) suggest that categorization would be affected by odorword pairing consistency because more consistent verbal cues will help make key perceptual features more salient and thereby enhance category formation. 
A growing number of studies (e.g., Barnhart, Rivera, \& Robinson, 2018; Lupyan, Rakison, \& McClelland, 2007; Lupyan \& Casasanto, 2015) show that initially meaningless words can draw attention to perceptual features across stimuli in a way that supports meaningful categorization. Lupyan and Casasanto (2015), following Lupyan et al. (2007), used a supervised categorization task to test whether verbal labeling has the power to transform mental representations of objects. Adult participants were asked to sort "aliens" as either approachable or unapproachable under three conditions. Aliens were co-presented with existing words ('round' vs. 'pointy') in a conventional-label condition, with pseudowords ('foove' vs. 'crelch') in a nonce-label condition, and without words in a control condition. The results showed that participants learned the relevant categorization equally well with existing words as they did with pseudowords, and that both groups with verbal labels outperformed participants who learned to categorize in the absence of labels.

One account of the facilitative effect of word-assisted categorization is that novel verbal labels, just like real-world labels, aid category learning because they simplify between-category distinctions by making the relevant perceptual dimensions (roundedness vs. pointiness) more concrete and thus easier to recall as the categorization task progresses. In other words, labels affect mental representations of categories by directing attention to key shared features of objects, and, in this way, enhance successful storage of category-defining visual features, as well as retrieval from memory in subsequent trials. The use of pseudowords alongside existing words helped to demonstrate that, rather than their specific content, the mere presence of verbal labels can scaffold category formation. However, the extent to which sound symbolism ('foove' more like 'round' vs. 'crelch' more like 'pointy'; e.g., Parise \& Spence, 2012) may have driven category formation remains questionable. Our study controls the possibility that labels may be associated to existing entries in the mental lexicon by systematically counterbalancing labels across participants. To further minimize potential effects of pre-existing lexical-semantic links, we chose smell, an optimal candidate from among the senses because of its low codability (i.e., low consensus in verbal labeling) across a range of unrelated languages, including English (Majid et al., 2018).

The vast majority of studies testing the effects of new labels on categorization have thus far relied on visual stimuli (e.g. Althaus \& Plunkett, 2016; Barnhart et al., 2018; Lupyan et al., 2007; Lupyan \& Casasanto, 2015), but there are exceptions. In a recent multisensory study that ventured outside the domain of vision, Miller, Schmidt, Blankenburg, and Pulvermüller (2019) tested whether associations formed between auditory linguistic labels and tactile stimuli had a facilitative effect on perceptual discrimination. Participants were copresented pseudowords with new tactile stimuli. Unbeknownst to the participants, the pseudoword tactile pairs were associated concordantly (70\% consistent) in one condition, and discordantly ( $25 \%$ consistent) in another condition. After a week of training, discrimination ability for tactile stimuli improved in the concordant but not discordant condition, even though stimulus frequencies and exposure length were kept the same.

An important feature of this paradigm was that learning the correct tactile pattern could happen entirely without linguistic labels. The observation that arbitrary labels irrelevant to the task assisted tactile stimulus discrimination was interpreted as evidence that language can affect perceptual discrimination through implicit associations formed between consistently paired linguistic and non-linguistic stimuli. In this study, we focus not on whether labels can assist perceptual discrimination of stimuli that are hard to differentiate, but on how much labels facilitate category formation where associations between new labels and hardto-identify percepts are implicitly formed in a perceptual learning context. We adopt Goldstone's (1998) definition of perceptual learning as changes to the perceptual system that improve the organism's ability to respond to the environment by increasing their 
attention to critical perceptual dimensions (1998: 585-588). While Miller et al. (2019) examined stimulus discrimination that taps into low-level perceptual processing, here we direct our focus of inquiry to how novel labels draw attention to perceptual features across different stimuli in ways that support category formation. We hypothesised that perceptual learning of categories will be more successful with consistent than with inconsistent labelpercept pairings.

We chose the domain of olfaction to test language effects on perceptual processing for four reasons. First, unlike vision or audition, the linguistic codability of smells tends to be more impoverished across very different language communities (Majid et al. 2018). This is an excellent design feature because it enables us to manipulate odor categories within subjects more freely than if there were strong pre-existing links between words and odors (see also Speed \& Majid, 2018). Second, a design where participants implicitly learn how a new label relates to odors, commonly known to represent "the muted sense" (Olofson \& Gotfried, 2015), enables us to compare category formation patterns when olfactory cues are "unmuted" either with concordantly or discordantly paired linguistic cues. Third, working with a modality where stimulus identification is difficult but trainable (Fournel, Sezille, Licon, Sinding, et al. 2017; Morquecho-Campos, Larsson, Boesveldt, Olofsson, 2019) helps avoid rapid ceiling effects. We can work with a modality where initial categorization performance is likely to be near chance level with gradual accuracy increases over learning. Ceiling effects are known to have complicated the interpretation of previous supervised visual categorization research (e.g., Kersten et al., 2010). Finally, we step outside visual modality into the olfactory modality because-unlike in vision-humans are largely limited to identifying only one odorant at a time (Stevenson \& Wilson, 2007; Yeshurun \& Sobel, 2010). This characteristic is ideal for a design with sequential ordering of single stimuli (widely known in categorization research as an ABX design). The observation that abstraction based on attending to what different odors have in common is possible, but rare (Majid \& Burenhult, 2014), helps us to discount potential effects of individual odor categorization habits. Pairing odors that are hard to define with labels that have no meaning puts us in a good position to compare whether increases in categorization accuracy are greater with more consistent odor labeling. If they are, it would demonstrate a causal link between language and olfactory perceptual processing that supports categorization.

\section{Materials and methods}

\subsection{Participants}

Forty-four English native speakers (age, $M=22.6, S D=3.4$, 29 female) participated in the study. The target sample size was 40 participants who met the inclusion criteria. Participants were randomly allocated to one of the two equally sized predetermined training groups. A sample size of 20 participants per group was deemed to have sufficient statistical power based on earlier related studies with comparable tasks (Dolscheid et al., 2013; Miller et al., 2018). The inclusion criteria were no cold or allergies, such as hayfever, affecting the sense of smell at the time of testing; no use of any psycho-active medication; no prior history of drug or alcohol abuse, neurological, psychiatric or sleep disorders; non-smoker; and no diagnosis of anosmia. We also tested for olfactory discrimination using the validated Sniffin' Sticks test (Hummel et al., 1997) and participants overall scored over $62.5 \%$ ( $\geq 10$ correct answers out of 16 ). Smell discrimination ranged from 10 to 16 (mean core $12.58 \pm 1.66 \mathrm{SD}$ ) 
and was comparable between the two experimental groups $(M=13.00, S D=1.62, M=$ $12.15, S D=1.63$ ). While between-participant variation was relatively low, the range of incorrect answers per odorant varied between 2/44 (anise) to 22/44 (rose). The three odorants that were most often incorrectly discriminated in both groups were rose (22/44), cinnamon (20/44) and garlic (18/44). Data from 4 participants were excluded from analyses, one due to low smell discrimination ability (9/16), two due to a recording malfunction, and one because the participant developed a cold in the training phase. Participation was remunerated, and the experiment lasted approximately 2.5 hours (30 mins of training daily across four consecutive days and a half-hour test on day four).

\subsection{Olfactory stimuli}

For the main experiment, eighteen odor pens, i.e., Sniffin' Sticks (Hummel et al., 1997, Rumeau et al., 2016) were arranged into six triplets. Each triplet consisted of two test odors ( $A$ and $B$ ) and one reference odor $(X)$. The reference odor was always presented last. The triplets (in ABX order) were leather-smoked meat-mushroom, eucalyptus-mint-grass, caramel-coconut-coke, peach-melon-raspberry, banana-pear-pineapple, and lilac-lavenderrose. These six odor triplets together formed one block. Each participant was exposed to four blocks a day, four days in a row. An additional odor triplet, lemon-coffee-orange, was used for task illustration purposes each day before the start of the first block. This was the only triplet presented with real-world odor labels. Triplets were constructed so as to differ less within a triplet (e.g., three floral scents in lilac-lavender-rose) and more across triplets (e.g., floral vs. fruity scents).

To ensure triplets were optimised for within-triplet similarity, a separate study with 20 native English speakers who did not participate in the main study was conducted.

Participants provided similarity ratings (scale 1-9: $1=$ very similar; $9=$ very different) of odor pairs $(50 \%$ in XA and $50 \%$ in XB order) to verify that perceptual distances between odor pairs within each triplet were comparable. The aim was to identify six triplets for which odors $A$ and $B$ were comparably similar to $X$. Twenty one odorants were used in this pilot test. Three odorants were excluded from the main test because they were perceived as unpleasant (fish, onion) or poorly discernible (grapefruit). The average ratings ordered by decreasing similarity of the closer pair were (rose-lilac $M=3.5, S D= \pm 1.76$, rose-lavender $M=4.3, S D=1.63$; raspberry-peach $M=3.6, S D=1.64$, raspberry-melon $M=3.8, S D=$ 1.97; mushroom-leather $M=4.7, S D=1.75$, mushroom-smoked meat $M=5.85, S D=2.03$; pineapple- banana $M=4.82, S D=1.7$, pineapple-pear $M=5.65, S D=1.69$; grass-mint $M=$ $5.92, S D=1.88$, grass-eucalyptus $M=6.7, S D=1.95$; caramel-coke $M=6.2, S D=2.14$, caramel-coconut $M=6.85, S D=1.9$ ). These six $A B X$ triplets were included in the main experiment as they met the criterion of no statistically significant $A X$ vs $B X$ difference in perceived similarity ( $p>.004$, Bonferroni-adjusted for multiple comparisons).

\subsection{Verbal stimuli}

Eighteen bisyllabic pseudowords conforming to English phonotactic rules were selected from an established collection (Rastle \& Colehart, 2000) to serve as verbal labels. The labels were hogger, dastude, zabnart, kabist, mootite, bitjeed, nurhact, holpbon, rodgeap, portak, vokine, chigor, jortle, pizlime, vebtaze, irsabe, wodment, melpow. This selection was 
motivated by the labels' absence of conventionalized meanings. The labels were read out and recorded by a male native English speaker, with stress on the initial syllable in each pseudoword. The presentation length across pseudowords was kept constant (2000 ms).

\subsection{Counterbalancing and randomization}

Four levels of randomization and counterbalancing were implemented. First, odor and label pairings were fully randomized and counterbalanced across participants to avoid potential stimulus-driven biases. Each participant was randomly assigned a unique combination of pseudowords and smells to control for the potential effect of pre-existing phono-semantic associations (Nygaard, Cook, \& Namy, 2009) and of sound-symbolism for smell (O'Meara, Kung, \& Majid, 2019). Example configurations are available on the project website at https://osf.io/kj5sc/. Second, the order of triplet presentation (ABX or BAX) varied across blocks. That is, for $50 \%$ of participants the order of triplet presentation in a four-block training session was $A B X-B A X-B A X-A B X$ and for the other $50 \%$ of participants the order-block configuration was $B A X-A B X-A B X-B A X$. Third, to further eliminate potential sequencing effects, the order of triplet presentation also varied within blocks. The six triplets were shuffled into an initial random order. This order was set to change using a latin-square design (e.g. if order $1=a b c d e f$ and order $2=b c d e f a$ for block 1 , then order $1=b c d e f a$ and order $2=$ cdefab for block 2 etc.). Finally, the assignment of the correct answer differed across participants. For $50 \%$ of participants in each training condition A was the correct answer (i.e., more similar to X), and for the other $50 \%$ B was correct.

\subsection{Training conditions}

Two training conditions were implemented to test the effect of verbal labeling on the ability to categorize olfactory stimuli. In the first training condition (CONCORD), participants performed the task with $81.25 \%$ consistent smell-label parings, and in the second condition (DISCORD), participants received training with only $25 \%$ consistent smell-label pairings (see Fig. 1). The task and the frequency of encountering each smell and each pseudoword were identical across the two training conditions. The only manipulation was in the high vs. low consistency of smell-label pairings. 
(b)
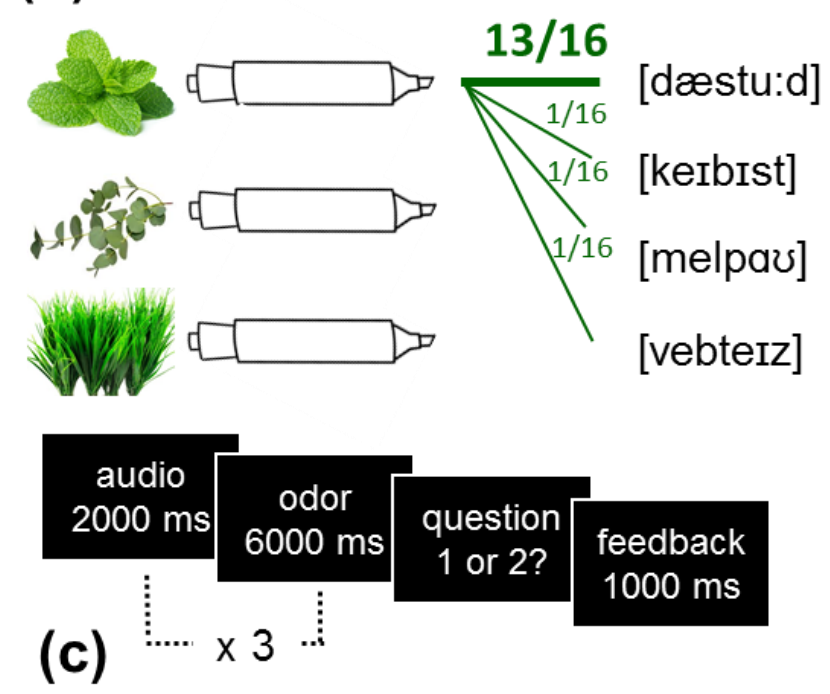

DISCORD

(a)

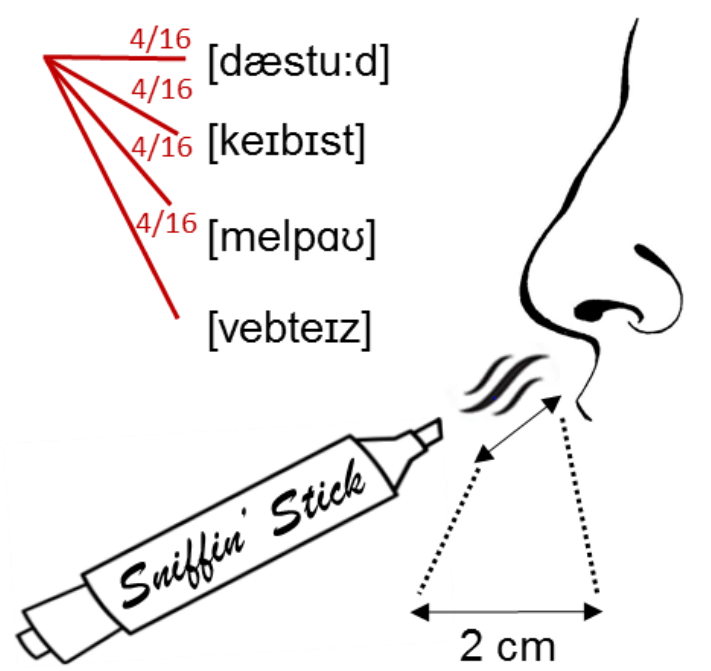

Fig. 1. Experiment design. (a) Sniffin' sticks were used as olfactory stimuli. They were presented in a quiet and well-ventilated room, on a static platform, using an odorless glove, with the tip of the pen held around 2 centimetres under the participant's nose. (b) Bisyllabic pseudowords used as verbal stimuli were co-presented with the odors. For the training phase, pseudoword pairs were presented under a consistent (CONCORD) or an inconsistent (DISCORD) condition. Each odor was presented 16 times in total, always within the same triplet. To manipulate the strength of associations formed during training, the consistency of odor-pseudoword pairs was $13 / 16$ in the CONCORD condition and 4/16 in the DISCORD condition (as shown for mint-odor within the minteucalyptus-grass odor triplet). (c) The odor similarity judgment test used the same task design as the first day of training, except that the test was performed without presentation of pseudowords.

\subsection{Procedure}

The experiment was programmed using Experiment Builder (SR Research, 2017, v 2.1.512). Participants first saw an instruction screen informing them that their task was to smell sets of three odors while listening to new words. The experimenter emphasized the importance of focusing on the odor of each pen and of not touching the tip of the pen with their nose or hands. After the third odor, participants indicated by pressing a button on a keyboard which odor, 1 or 2, was most similar to the third odor. Once they had made their selection of 1 or 2 , a green tick appeared if their choice was correct, and a red cross if incorrect. They were asked to pay attention to all three aspects of the task, i.e. the odors, the language labels, and the feedback.

In line with recent reports on phase synchrony required for olfactory-auditory integration (Zhou et al., 2019), each trial began with an audio-recorded label (2 s), followed by presentation of an odor A (6s). Subsequently, participants heard a different label (2s) before odor B ( $6 \mathrm{~s})$, and then another label ( $2 \mathrm{~s})$ before odor X (6s). Next, a question appeared asking whether A or B was more similar to X (until keypress). Feedback on accuracy (1s) was given at the end of each trial (1 s) (Fig. 1).

One triplet comprised three similar smells co-presented with three different pseudowords. Following previous perceptual learning studies (Athanasopoulos \& Albright, 2016; Vanek, 2019), each triplet was presented four times; twice in ABX and twice in BAX order. Every participant was trained on 96 triplets in total. 
Each participant was presented with four blocks of learning trials daily (approximately 30 minutes) for four consecutive days. One block consisted of 6 odor triplets and took approximately 7 minutes to complete. After each block, participants were given a short break to rest (terminated via a key press) and were shown the number of correct responses they scored out of 6 for that block. No explicit information about categorization rules was provided.

\subsection{Smell similarity judgment test}

After four days of training, participants completed an odor similarity judgement test to provide a measure of change in their odor categorization accuracy. Unlike the training phase, the test did not include verbal labels. Each participant was tested using the same four blocks of odor combinations they had been exposed to during their first day (i.e. Session 1) so that accuracy changes over time were directly comparable. A point was earned for each correct response, just as during training. After each block, participants were shown how many correct responses they had. The test took approximately 30 minutes.

In line with previous categorization studies with feedback (Kersten et al., 2010; Athanasopoulos \& Albright, 2016, Vanek, 2019), the measured variable was the response accuracy per participant per block. We predicted that concordant labeling would lead to higher categorization accuracy than discordant labeling because it would facilitate access to key perceptual features of odors in subsequent trials.

\section{Results}

\subsection{Learning Gains}

To measure changes in odor categorization accuracy after the training phase, for each participant we calculated their learning gains (\% correct in the test minus \% correct in Session 1). This approach allowed us to compare changes in odor categorization between training conditions (concordant vs. discordant). In Session 1, there was no difference in categorization accuracy between the two conditions $\left(M_{c o N}=50.00 \%, S D=12.63, M_{D I S}=\right.$ $49.79 \%, S D=14.46)$. However, after the training phase in the test without verbal labels, the concordant group outperformed the discordant group ( $M_{c o n}=74.17 \%, S D=13.38, M_{\text {ols }}=$ $61.88 \%, S D=15.83)$. Learning gains were larger in the concordant group $(M=24.17 \%, S D$ $=17.08)$ than the discordant group $(M=12.09, S D=18.33)$; see Fig. 2a. Increases in categorization accuracy were not correlated with participants' odor discrimination ability ( $p$ $>.5$ for both groups); Fig $2 b$. 

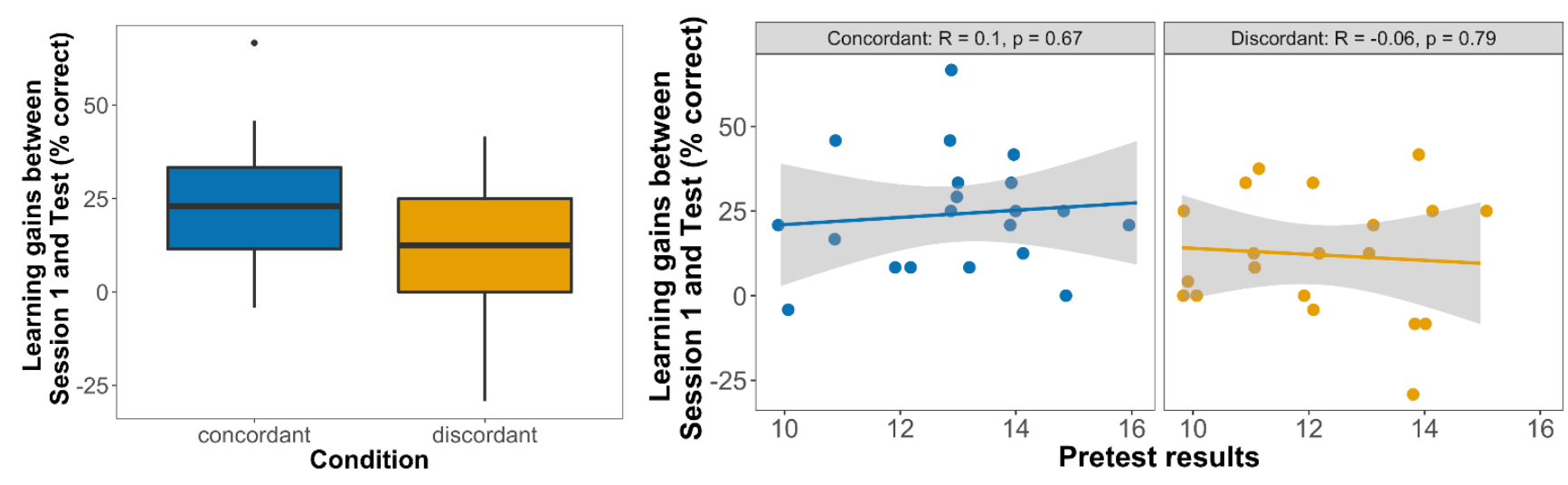

Fig. 2. (a) Learning gains in odor categorization in concordant vs. discordant training context. These gains were calculated by deducting participants' Session 1 score from their Test score after training. The final test was performed without verbal labels to examine the strength of associations formed during the two types of training. (b) Correlation between participants' pretest odor discrimination ability and their learning gains in the concordant and discordant groups. There was no inadvertent difference in discrimination ability across groups that can account for superior categorization accuracy of the concordant group.

To examine the relationships between training condition and odor categorization accuracy, we fit a logistic mixed effects model to a subset of the data including responses only from Session 1 (on day 1 ) and the Test (on day 4) using R (R Core Team, 2016) and Ime4 (Baayen, Davidson, \& Bates, 2008; Bates, Maechler, Bolker, \& Walker, 2015). This model is a direct confirmatory test of our main hypothesis that the learning of odor categories is aided by concordant labeling. The outcome variable of this model is response accuracy, coded as a binary variable (accurate vs. inaccurate). Training condition (concordant vs. discordant labeling), session (Session 1 vs. Test) and their interaction were entered as fixed effect variables. The model included all possible random effects (Barr, Levy, Scheepers, \& Tily, 2013): random slopes over session by participant and random slopes over session, condition and their interaction by item. The Ime4 formula used to fit the model is as follows:

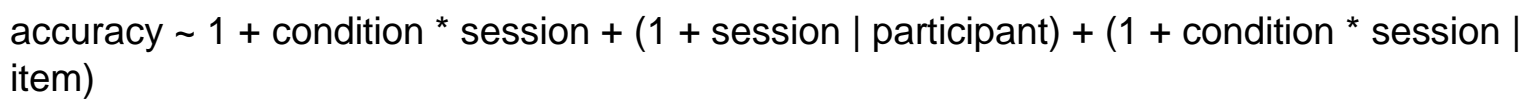

The model confirms our hypothesis: there is a significant interaction between condition and session $(\beta=-0.60, z=-2.25, p=0.025)$. The negative sign of this interaction suggests that participants in the discordant condition showed less of an improvement in accuracy than participants in the concordant condition. Predictions from the fitted model suggest that in the discordant condition, accuracy improved from $50 \%$ to $63 \%$ between Session 1 and Test session; while in the concordant condition it improved from $50 \%$ to $75 \%$. The full model output is available on the project website https://osf.io/ki5sc/. To summarize, the analyses confirmed the prediction that concordant labeling leads to higher categorization accuracy than discordant labeling.

\subsection{Learning Trajectories}

The second step of the analysis was a comparison of the overall rate of change. We ask how categorization patterns change with training over four session of training and the test session. As accuracy scores in a perceptual learning task are potentially nonlinear over time, 
we used generalized additive mixed modelling (GAMM) (Baayen, Vasishth, Kliegl \& Bates, 2017; Sóskuthy, 2017) to capture session-by-session changes in categorization.
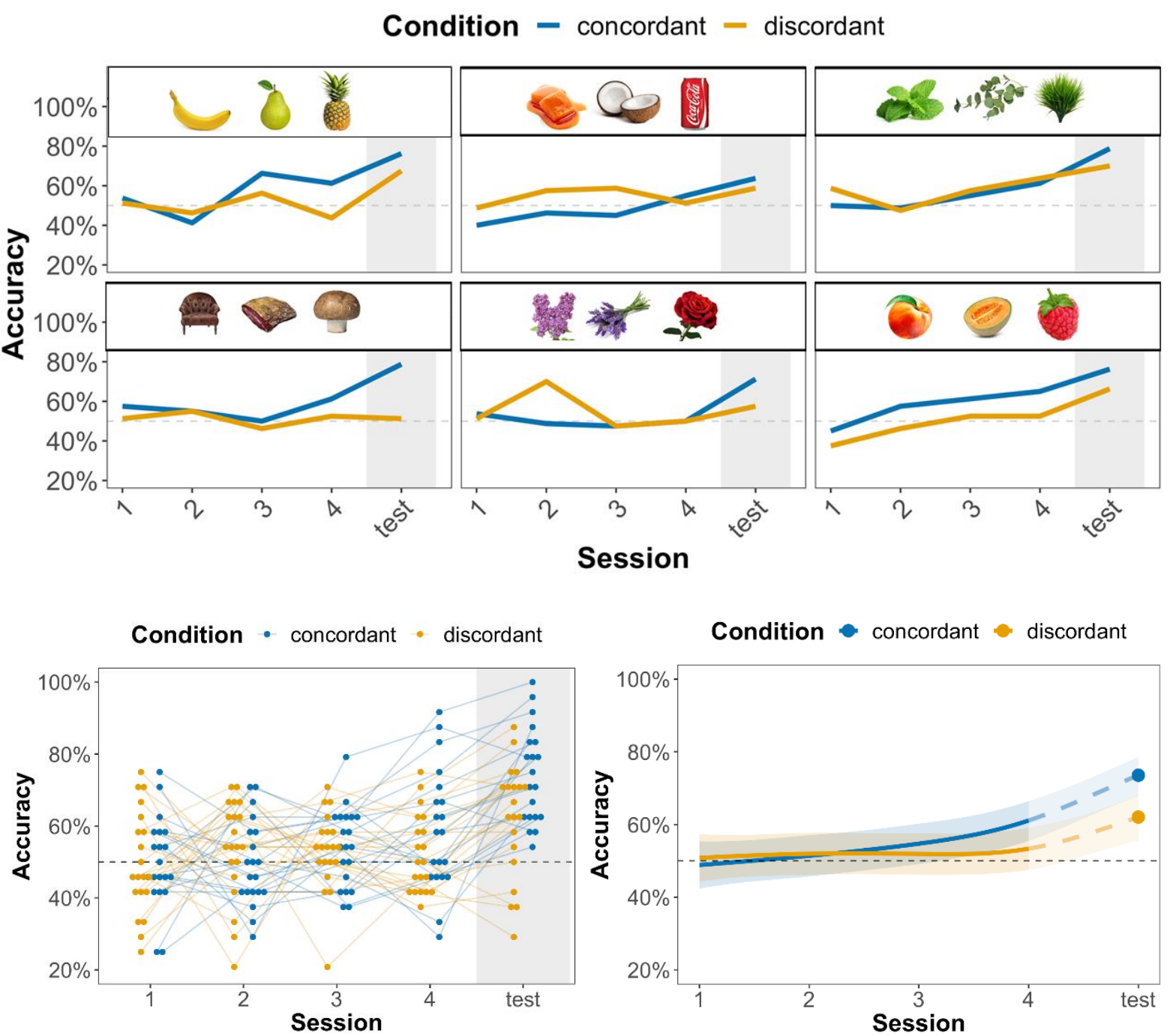

Fig. 3. (a) Changes in categorization accuracy (vertical axis) across the four training sessions and Test session (shown along the horizontal axis) for each odor triplet. Average accuracy is shown for participants in concordant (blue) and discordant (orange) conditions. In the Test session, unlike the training sessions, categorization was performed without verbal labels. The dashed horizontal line indicates baseline, i.e., chance performance, 50\%. The results for the six odor triplets are shown in the following order (from right to left, top row) banana-pearpineapple, caramel-coconut-coke, eucalyptus-mint-grass, (bottom row) leather-smoked meat-mushroom, lilaclavender-rose, peach-melon-raspberry. (b) Changes in categorization accuracy per subject, with each line representing an individual participant. (c) Changes in categorization accuracy according to the generalised additive mixed model. Lines show predicted accuracy, with 95\% confidence intervals indicated in grey. Lines are based on interpolation and are continuous to aid interpretation.

In an exploratory analysis we investigated the trajectory of category learning over time. Figure $3 a$ shows changes in categorization accuracy for each triplet/item. While there is variation across items, general trends are observable. Items improve in categorization accuracy over time; and participants in the concordant condition finish with higher categorization accuracy than their counterparts in the discordant condition. Some triplets such as grass-mint-eucalyptus, raspberry-peach-melon and (to a lesser extent) pineapple- 
banana-pear show continuous improvement over the training and test sessions, while others such as rose-lilac-lavender and mushroom-smoked meat-leather show an abrupt increase in accuracy towards the end. Figure $3 b$ shows the same changes grouped by participants, and reveals variation across individuals. While some participants' categorization accuracy does not improve, others show improvement, with more individuals above the $50 \%$ chance level than below. Figure $3 \mathrm{c}$ shows smoothed model predictions from a generalised additive mixed model (Wood 2017; Winter \& Wieling, 2016, Sóskuthy 2017). The statistical model underlying this plot controls for dependencies within participants and items, and generates learning curve estimates that are no more wiggly than justified by the underlying data (see Sóskuthy 2017 for more detail). Our GAMM predictions indicate that categorization accuracy rises steadily from the third session in the concordant condition (in \% correct, $M_{s 1}=50.00$, $S D=12.61 ; M_{s 2}=49.58, S D=11.93 ; M_{s 3}=54.17, S D=10.98 ; M_{s 4}=58.96, S D=17.01$, Test $M=74.17, S D=13.36)$, but only starts to improve later, at the test stage, in the discordant condition $\left(M_{s 1}=49.79, S D=14.46 ; M_{s 2}=53.75, S D=13.91 ; M_{s 3}=53.13, S D=\right.$ 10.89; $\left.M_{S 4}=52.29, S D=10.94, M_{T E S T}=61.88, S D=15.84\right)$. This difference between the two learning curves is statistically significant according to a chi-squared test on the smooth corresponding to the estimated difference between the conditions (estimated $d f=1.678 ; \chi^{2}=$ 7.224; $p=0.036)$.

\section{Discussion}

We investigated if simply hearing arbitrary linguistic labels that are concordantly or discordantly paired with olfactory stimuli benefits olfactory category learning in adults. We found participants learned new categories with more success when there was a consistent dominant label paired with an odor. Inconsistent pairing of labels to odors did not prevent categorization learning, but it certainly reduced overall learning gains as well as learning speed. While attention optimization during category learning with novel labels (Lupyan et al., 2007) and with feedback (Blair, Watson, \& Meier, 2009) are well documented, this is the first study to report labeling effects on category formation in the olfactory modality using differential linguistic context. These findings are striking in that they suggest that odor categorization may be better in linguistic communities with more consistent odor labelling (e.g., the hunter-gatherer Jahai) than in linguistic communities with inconsistent odor labelling (i.e., English-speakers; cf. Majid \& Burenhult, 2014).

\subsection{Category formation enhanced by concordant labels}

We predicted that labels paired with odors in a concordant manner would direct attention to key perceptual features of odors, making them more concrete, and thus aiding their categorization. The results are consistent with this reasoning by showing that concordant labeling significantly enhanced categorization accuracy in comparison to discordant labeling. Such enhancement in forming olfactory categories can be explained by perceptual learning, possibly through a mechanism in which language can tune perception by selectively directing attention to specific perceptual attributes (Goldstone, 1998, Smith, Jones, Landau, Gerschfoff-Stowe, \& Samuelson, 2002). With repeated training, odor attributes (for instance, similarity in odor quality) that are relevant to form a category become selectively attended to while others (differences in odor quality) get selectively ignored. An alternative potential mechanism is that olfactory categories are formed through attribute differentiation 
(Goldstone \& Byrge, 2015), which, unlike attentional tuning, would assume that perceptual attributes do not start off as perceptually distinct, but only became separable through training. Under this view, training enabled modification of the perceptual system through weighing one attribute and learning to selectively attend to it while ignoring another.

Previous studies examining the effects of labels on perception have been met with skepticism, raising concerns about lack of control over pre-existing links between linguistic and perceptual stimuli (e.g., Boutonnet et al., 2013). Here, we minimised this risk by using unfamiliar pseudowords and odors that do not have strong prior links to linguistic labels in our recruited group of speakers (i.e., English speakers who when asked to freely name such odors perform poorly; cf. Majid \& Burenhult, 2014). However, one of the questions that remains is whether the facilitative effect of concordant labeling of smells emerged as a result of increasing their perceived similarity (e.g., Sloutsky, Lo, \& Fisher, 2001) or directing attention to their salient distinguishing features and making those more salient (Lupyan \& Casasanto, 2015). After training, participants did not report any specific strategy. To address this question, future research would benefit from adding a test on whether the degree of change in perceived odor similarity before and after training can predict learning gains.

\subsection{Labeling effects and linguistic relativity}

There are two points of criticism generated by previous studies on supervised categorization which the present study avoids. Skeptics may argue that claiming language-driven effects based on differences in performance when one group categorizes with and the other without labels (e.g., Kersten et al., 2010; Vanek, 2019) can be problematic because of unmatched task complexity across groups. Another point of concern is that perceptual learning designs proceed without an initial task-independent check of general discrimination ability in the tested sensory domain (e.g., Miller et al., 2019), leaving open the possibility for individual variation to play a role. This study matched task complexity by presenting label-odor pairs to both groups, and tested odor discrimination ability to minimise the likelihood that the observed effects would be due to such confounds.

We showed that training with labels affected how people categorized odors in a test without labels. We interpret this to show that language can play a causal role in shaping perceptual processes that drive category formation. This is likely to stem from mapping new verbal labels onto odors, a process where attention gets optimized so as to improve successful storage of category-defining perceptual features (Lupyan et al., 2007) and reduce error as categorization with feedback unfolds (Blair et al., 2009). The non-linguistic odor categorization test at the end of the learning sessions, suggest that the changes go beyond mere overt labeling to shaping the odor categories themselves. This suggests a sustained effect on how linguistically labelled smells are perceived and categorized. Future studies could unpack how deep these effects go by using verbal interference tasks, for example, in the test phase, to establish definitively whether odor perception has been modulated. 


\subsection{Conclusion}

Effects of labeling on category formation are well established, but studies exploring their underlying mechanisms have brought mixed results and have been open to different interpretations. In this study, the presence of meaningless pseudowords enhanced the formation of arbitrary odor categories when pseudowords and odor stimuli were consistently paired. Learning gains were present but delayed and significantly reduced with inconsistent pseudoword-odor pairs. These findings bring two new insights to previous research on labeling effects. First, they demonstrate that new verbal cues undergo multisensory integration with co-presented odors and affect the capacity to categorize olfactory input. And second, they demonstrate that consistent language input aids olfactory perceptual processing and improves expertise in odor categories markedly faster than inconsistent input. The results show that different types of linguistic input (i.e., consistency of odor labelling) can influence category formation in substantially different ways, raising the possibility that not just naming, but the underlying conceptual representation of odors may differ across cultures in accordance with the language people speak.

\section{References}

Althaus, N., \& Plunkett, K. (2016). Categorization in infancy: labeling induces a persisting focus on commonalities. Developmental Science, 19(5), 770-780.

https://doi.org/10.1111/desc. 12358

Athanasopoulos, P., Bylund, E., Montero-Melis, G., Damjanovic, L., Schartner, A., Kibbe, A., ... \& Thierry, G. (2015). Two languages, two minds: Flexible cognitive processing driven by language of operation. Psychological Science, 26(4), 518-526.

https://doi.org/10.1177/0956797614567509

Athanasopoulos, P., \& Albright, D. (2016). A perceptual learning approach to the Whorfian hypothesis: Supervised classification of motion. Language Learning, 66(3), 666-689. https://doi.org/10.1111/lang.12180

Baayen, R. H., Davidson, D. J., \& Bates, D. M. (2008). Mixed-effects modeling with crossed random effects for subjects and items. Journal of Memory and Language, 59, 390-412. https://doi.org/10.1016/j.jml.2007.12.005

Baayen, R. H., Vasishth, S., Kliegl, R. and Bates, D. (2017). The cave of shadows:

Addressing the human factor with generalized additive mixed models. Journal of Memory and Language, 94, 206-234. https://doi.org/10.1016/j.jml.2016.11.006

Barnhart, W. R., Rivera, S., \& Robinson, C. W. (2018). Effects of linguistic labels on visual attention in children and young adults. Frontiers in Psychology, 9, 358.

https://doi.org/10.3389/fpsyg.2018.00358

Barr, D. J., Levy, R., Scheepers, C., \& Tily, H. J. (2013). Random effects structure for confirmatory hypothesis testing: Keep it maximal. Journal of Memory and Language, 68, 255-278. https://doi.org/10.1016/.j.jml.2012.11.001

Bates, D., Maechler, M., Bolker, B., \& Walker, S. (2015). Fitting linear mixed-effects models using Ime4. Journal of Statistical Software, 67, 1-48. https://doi.org/10.18637/jss.v067.i01 
Boroditsky, L. (2001). Does language shape thought?: Mandarin and English speakers' conceptions of time. Cognitive Psychology, 43(1), 1-22.

https://doi.org/10.1006/cogp.2001.0748

Boutonnet, B., Dering, B., Viñas-Guasch, N., \& Thierry, G. (2013). Seeing objects through the language glass. Journal of Cognitive Neuroscience, 25(10), 1702-1710.

https://doi.org/10.1162/jocn a 00415

Dolscheid, S., Shayan, S., Majid, A., \& Casasanto, D. (2013). The thickness of musical pitch: Psychophysical evidence for linguistic relativity. Psychological Science, 24(5), 613-621, https://doi.org/10.1177/0956797612457374

Fournel, A., Sezille, C., Licon, C. C., Sinding, C., Gerber, J., Ferdenzi, C., Hummel. T., \& Bensafi, M. (2017). Learning to name smells increases activity in heteromodal semantic areas. Human brain mapping, 38(12), 5958-5969. https://doi.org/10.1002/hbm.23801

Franklin, A., Pilling, M., \& Davies, I. (2005). The nature of infant color categorization: Evidence from eye movements on a target detection task. Journal of Experimental Child Psychology, 91(3), 227-248. https://doi.org/10.1016/i.jecp.2005.03.003

Freundlieb, N., Ridder, V., Dobel, C., Enriquez-Geppert, S., Baumgaertner, A., Zwitserlood, P., ... \& Liuzzi, G. (2012). Associative vocabulary learning: development and testing of two paradigms for the (re-) acquisition of action-and object-related words. PloS One, 7(6). https://doi.org/10.1371/journal.pone.0037033

Gleitman, L., \& Papafragou, A. (2005). Language and thought. Cambridge Handbook of Thinking and Reasoning, 633-661. Cambridge: Cambridge University Press.

Goldstone, L. (1998). Perceptual learning. Annual Review of Psychology, 49, 585-612. https://doi.org/10.1146/annurev.psych.49.1.585

Goldstone, R. L., \& Byrge, L. A. (2015). Perceptual learning. Oxford Handbook for Philosophy of Perception. Oxford: Oxford University Press.

Hummel, T., Sekinger, B., Wolf, S., Pauli, E., Kobal, G., (1997). 'Sniffin' Sticks': Olfactory performance assessed by the combined testing of odor identification, odor discrimination and olfactory threshold, Chemical Senses, 22(1), 39-52.

https://doi.org/10.1093/chemse/22.1.39

Jackendoff, R. (1990). Semantic structures. Cambridge, MA: MIT Press.

Kersten, A. W., Meissner, C. A., Lechuga, J., Schwartz, B. L., Albrechtsen, J. S., \& Iglesias, A. (2010). English speakers attend more strongly than Spanish speakers to manner of motion when classifying novel objects and events. Journal of Experimental Psychology: General, 139(4), 638-653. https://doi.org/10.1037/a0020507

Levinson, S., \& Bowerman, M. (2001). Language acquisition and conceptual development. No. 3. Cambridge: Cambridge University Press.

Lupyan, G., Rakison, D. H., \& McClelland, J. L. (2007). Language is not just for talking: Redundant labels facilitate learning of novel categories. Psychological Science, 18(12), 1077-1083. https://doi.org/10.1111/j.1467-9280.2007.02028.x

Lupyan, G., \& Casasanto, D. (2015). Meaningless words promote meaningful categorization. Language and Cognition, 7(2), 167-193. https://doi.org/10.1017/langcog.2014.21 
Majid, A., Roberts, S. G., Cilissen, L., Emmorey, K., Nicodemus, B., O’Grady, L., [...] \& Shayan, S. (2018). Differential coding of perception in the world's languages. Proceedings of the National Academy of Sciences, 115(45), 11369-11376.

https://doi.org/10.1073/pnas.1720419115

Majid, A., \& Burenhult, N. (2014). Odors are expressible in language, as long as you speak the right language. Cognition, 130(2), 266-270.

https://doi.org/10.1016/i.cognition.2013.11.004

Malt, B. C., Sloman, S. A., Gennari, S., Shi, M., \& Wang, Y. (1999). Knowing versus naming: Similarity and the linguistic categorization of artifacts. Journal of Memory and Language, 40(2), 230-262. https://doi.org/10.1006/imla.1998.2593

Morquecho-Campos, P., Larsson, M., Boesveldt, S., \& Olofsson, J. K. (2019). Achieving olfactory expertise: Training for transfer in odor identification, Chemical Senses, 1-7.

https://doi.org/10.1093/chemse/bjz007

Miller, T., Schmidt, T., Blankenburg, F., \& Pulvermüller, F. (2018). Verbal labels facilitate tactile perception. Cognition, 171, 172-179. https://doi.org/10.1016/i.cognition.2017.10.010

Nygaard, L., Cook, A., \& Namy, L. (2009). Sound to meaning correspondences facilitate word learning. Cognition, 112(1), 181-186.

https://dx.doi.org/10.1016/i.cognition.2009.04.001

Olofsson, J. K., \& Gottfried, J. A. (2015). The muted sense: neurocognitive limitations of olfactory language. Trends in Cognitive Sciences, 19(6), 314-321.

https://doi.org/10.1016/i.tics.2015.04.007

O'Meara, C., Kung, S., \& Majid, A. (2019). The challenge of olfactory ideophones:

Reconsidering ineffability from the Totonac-Tepehua perspective. International Journal of American Linguistics, 85(2), 173-212. https://doi.org/10.1086/701801

Papafragou, A., Hulbert, J., \& Trueswell, J. (2008). Does language guide event perception? Evidence from eye movements. Cognition, 108(1), 155-184.

https://doi.org/10.1016/i.cognition.2008.02.007

Parise, C. V., \& Spence, C. (2012). Audiovisual crossmodal correspondences and sound symbolism: a study using the implicit association test. Experimental Brain Research, 220(34), 319-333. https://doi.org/10.1007/s00221-012-3140-6

Rastle, K. \& Coleheart, M. (2000). Lexical and non-lexical print-to-sound translation of disyllabic words and non-words. Journal of Memory and Language, 42, 342-364.

https://doi:10.1006/imla.1999.2687

Rumeau, C., Nguyen, D., \& Jankowski, R. (2016). How to assess olfactory performance with the Sniffin' Sticks test ${ }$. European Annals of Otorhinolaryngology, Head and Neck Diseases, 133(3), 203-206._https://doi.org/10.1016/j.anorl.2015.08.004

Sloutsky, V. M., Lo, Y. F., \& Fisher, A. V. (2001). How much does a shared name make things similar? Linguistic labels, similarity, and the development of inductive inference. Child Development, 72(6), 1695-1709. https://doi.org/10.1111/1467-8624.00373

Smith, L.B., Jones, S. S., Landau, B., Gershkoff-Stowe, L., \& Samuelson, L. (2002) Object name learning provides on-the-job training for attention. Psychological Science, 13(1), 1319. https://doi.org/10.1111/1467-9280.00403 
Sóskuthy, M. (2017). Generalised additive mixed models for dynamic analysis in linguistics: a practical introduction. arXiv:1703.05339 [stat: AP].

Stevenson, R. J., \& Wilson, D. A. (2007). Odour perception: an object-recognition approach. Perception, 36(12), 1821-1833. https://doi.org/10.1068/p5563

Thierry, G., Athanasopoulos, P., Wiggett, A., Dering, B., \& Kuipers, J. R. (2009). Unconscious effects of language-specific terminology on preattentive color perception. Proceedings of the National Academy of Sciences, 106(11), 4567-4570. https://doi.org/10.1073/pnas.0811155106

Vanek, N. (2019). Changing event categorization in second language users through perceptual learning. Language Learning. https://doi.org/10.1111/lang.12377

Waxman, S. R., \& Markow, D. B. (1995). Words as invitations to form categories: Evidence from 12-to 13-month-old infants. Cognitive Psychology, 29(3), 257-302.

https://doi.org/10.1006/cogp.1995.1016

Winter, B., Wieling, M., 2016. How to analyze linguistic change using mixed models, Growth Curve Analysis and Generalized Additive Modeling. Journal of Language Evolution 1 (1), $7-$ 18. https://doi.org/10.1093/jole/lzv003

Wood, S., 2017. Generalized Additive Models: An Introduction with R, 2nd Edition. CRC Press, Boca Raton.

Yeshurun, Y., \& Sobel, N. (2010). An odor is not worth a thousand words: from multidimensional odors to unidimensional odor objects. Annual Review of Psychology, 61, 219-241. https://doi.org/10.1146/annurev.psych.60.110707.163639

Zhou, G., Lane, G., Noto, T., Arabkheradmand, G., Gottfried, J. A., Schuele, S. U., Rosenow, J. M., Olofson, J. K., Wilson, A. W., \& Zelano, C. (2019). Human olfactory-auditory integration requires phase synchrony between sensory cortices. Nature communications, 10(1), 1-12. https://doi.org/10.1038/s41467-019-09091-3 